\title{
In reply to Tin et al.
}

\author{
Gianluca Pellino • Francesco Selvaggi
}

Accepted: 8 January 2015 / Published online: 20 January 2015

(C) Springer-Verlag Berlin Heidelberg 2015

\section{Dear Editor:}

We read with interest the letter by Tin et al. [1] commenting on our paper dealing with a score to predict pain after excisional hemorrhoidectomy [2], recently published in the International Journal of Colorectal Disease. We thank the authors for their observations, which offered us the opportunity of giving some explanations.

Concerning the two scores to predict postoperative pain suggested by Tin et al., we did not compare our score with these for different reasons. First, only one is dealing with early-onset high pain after surgery (Boselli et al.), whereas the other one is focused on chronic pain (Althaus et al.). As clearly stated in our manuscript, we did not develop a score to predict prolonged pain, due to the limited number of observed events [2]. Second, one should consider that these scores are developed on a heterogeneous group of patients undergoing different types of surgical procedures, e.g., only 11 out of 245 patients $(4.5 \%)$ received surgery for abdominal diseases in one paper (Althaus et al.). Pain can occur after any type of surgery, but it is the most common complication after hemorrhoid surgery [2], and it is perceived as a major issue by patients with piles. In addition, the psychological distress which may be observed in patients diagnosed with malignancies, undergoing multimodal treatments or repeated surgery [3] might play a role in postoperative pain perception, making it difficult to obtain reasonable comparisons. This also leads us to exclude from our model patients undergoing procedures that were more complex than pure excisional hemorrhoidectomy [2].

Regarding the process of validation used for our score, it is not dissimilar from that reported in most studies aimed at developing predictive scores. As we clearly stated [2], sample size calculations were made before developing the score, and discrimination as well as goodness-of-fit were tested by means of accepted methods [2]. Furthermore, we provided a figure comparing directly the reliability of the score in development and validation group. Besides, an external validation would be desirable and likely to come to the point [4].

\section{References}

1. Tin SS, Wiwanitkit V (2014) Score to predict pain after excisional hemorrhoidectomy. Int J Colorectal Dis. doi: 10.1007/s00384-0142022-8

2. Selvaggi F, Pellino G, Sciaudone G, Candilio G, Canonico S (2014) Development and validation of a practical score to predict pain after excisional hemorrhoidectomy. Int J Colorectal Dis 29:1401-10

3. Selvaggi F, Fucini C, Pellino G, Sciaudone G, Maretto I, Mondi I, Bartolini N, Caminati F, Pucciarelli S (2014) Outcome and prognostic factors of local recurrent rectal cancer: a pooled analysis of $150 \mathrm{pa}-$ tients. Tech Coloproctol. doi: 10.1007/s10151-014-1241-x

4. Siontis GC, Tzoulaki I, Castaldi PJ, Ioannidis JP (2015) External validation of new risk prediction models is infrequent and reveals worse prognostic discrimination. J Clin Epidemiol 68:25-34
G. Pellino $\cdot$ F. Selvaggi $(\bowtie)$

Unit of General Surgery, Second University of Naples,

Via F. Giordani, 42, 80122 Naples, Italy

e-mail: fselvaggi@hotmail.com 